\title{
Design and Implementation of Intelligent Home Control System under the Concept of IoT
}

\author{
Weiliang $\mathrm{Li}^{1}$, Hao $\mathrm{Yu}^{2}$, Yantong Dou ${ }^{3}$ \\ ${ }^{1}$ HoHai University, Changzhou, 213022, China \\ ${ }^{2}$ Michigan State University, East Lansing, 48824, United States of America \\ ${ }^{3}$ Beijing University of Technology, Beijing, 100124, China
}

Keywords: Intelligent Home, Smart home, Control system, Internet of Things

\begin{abstract}
With the development and application of science and technology, smart home technology has gradually entered and improved people's traditional way of life, bringing great convenience and security for people's lives. Based on the analysis of the characteristics of smart home technology, this paper presents the design and implementation of smart home control system under the concept of Internet of Things. Practice has proved that the intelligent home control system based on the concept of Internet of Things realizes remote control of home equipment, and improves the convenience, comfort and security of people's lives.
\end{abstract}

\section{Introduction}

With the development of human society, computer technology, embedded technology, wireless communication technology has become an indispensable modern technology. In recent years, the Internet of Things has become the focus of global attention, and is considered as the most important technological innovation after the Internet. The Internet of Things transmits information between objects and objects, and between objects and the Internet through ZigBee, RFID, GSM/GPRS, infrared sensors and other information sensing devices in accordance with the agreed agreements. Intelligent home system based on Internet of Things is a kind of real-time monitoring and management system, which consists of intelligent home control management system, terminal (home sensor terminal, controller), home network, extranet network, information center. In the development and application of smart home, the combination of smart home and Internet of Things technology is an important development direction of smart home development and application. Subsequently, the domestic developed cities have emerged smart home research and development enterprises, smart home into the pioneering period. Then, because of the uneven development of smart home industry and other reasons, the whole smart home industry entered a period of hovering, some smart home companies out of the market. In recent years, leading enterprises have upgraded their intelligence to a strategic level. With the advent of new technologies and increasing recognition of smart home systems, smart home has entered a period of rapid development. In this paper, we will make full use of the Internet of Things to link the various layers of smart home system together to achieve information exchange between the various layers of smart home system to achieve the interactive control of people and things, things and things in smart home system [2].

\section{Design of Intelligent Home Control System under the Concept of IoT}

\subsection{Overall Design}

Smart home focuses on meeting the user's convenience and comfort needs, while reducing the development and maintenance costs as much as possible, convenient for later use and maintenance. This paper mainly uses Arduino hardware platform, Web server technology, HTTP communication technology, Android technology and so on. Users select different nodes through the remote control, 
the selected node LED light to prompt the user that the node is currently selected node, the user can press different buttons on the remote control, the device connected to the node to do the required operation. Users can adjust the brightness of the lamp, turn on or off the fan, and open or close the curtain by remote control. A remote controller can control different nodes at the same time, and a node can connect different devices at the same time. The specific working process of the system is as follows: the controller collects the home environment data by a series of sensors, then uploads the home environment data to the server through the wireless module via the WIFI network of the wireless router, and accepts the various instructions sent by the server, which are handed over to the executing components; on the other hand, the user can use the wireless module to upload the home environment data to the server through the WIFI network of It monitors the home environment through browser access server, and sends instructions to networked devices. At the same time, users can also use Android mobile phone APP to monitor the home environment anytime and anywhere. With the help of home gateway, users can use the computer to carry out remote operation or indoor remote monitoring of various household appliances through Ethernet. When it is inconvenient for users to use computers, they can also realize the remote monitoring of home through GPRS wireless network through smart phones. The system also has a friendly graphical user interface. The interface can display the running information of the components in the system. Users can conveniently query the running status of domestic devices [3].

\subsection{Hardware Design}

The hardware of the system is modular design. Modular design divides the system according to the function and designs the program with the function block as the unit. Modular design can reduce the complexity of the program, make the system structure clearer, and is more beneficial to the debugging and maintenance of the hardware system. The whole hardware system is divided into five modules, the first is the main control module, this module is the core of the hardware system, the control function of the whole system is achieved by the main control module, the core of the main control module is the STM32 microcontroller. Secondly, the power module, the entire hardware system needs power supply to work, so the power module is needed to power the entire system. Thirdly, the signal acquisition module, the system uses different types of sensors to monitor different parameters in the environment, so we need to design the signal acquisition module to monitor the parameters in the environment. Then, the drive module, intelligent control hardware such as bulbs, motors, need to be driven by the drive module to work, humidifier through the relay module to achieve switch control. Finally, the wireless WIFI transmission module, this wireless WIFI transmission module and the master STM32 microcontroller are connected by serial port, the wireless signal is converted into serial port signal, to achieve data transmission in the wireless network. The MCU needs to be initialized as needed before it is formally worked. Initialization mainly includes variables or functions used in some processes [4].

The main variables used in the system are stored temperature and humidity value, methane concentration value of the variables, switch statements in the marker, and so on. The functions to be initialized include delay function, interrupt function initialization, WIFI module serial port initialization function, development board LED lamp initialization, timer initialization and temperature and humidity sensor function initialization. SCM and sensor in data acquisition and data transmission time is in accordance with a certain sequence, which needs to cooperate with the delay function; interruption is the most commonly used mode of SCM, WIFI module receives messages through the interrupt mode to the SCM for processing, so the interruption function needs to be carried out. Initialization; WIFI module communicates with MCU through serial port. The logical relationship between WIFI module and MCU is that WIFI module sends messages, MCU receives messages, and vice versa. Therefore, the power supply of the WIFI module and the ground are connected to the power supply of the single-chip microcomputer respectively, and the power supply of the WIFI module is realized. The peripheral of the interface of the single-chip microcomputer is marked with the need to connect the sending end or the receiving end of the WIFI module. Lighting is the most commonly used function in family life. The lighting module of this system can mainly 
realize the lighting on and off and brightness adjustment.

In this system, the brightness adjustment is controlled by PWM (Pulse Width Modulation). PWM adjusts the principle of light by keeping the current steady through the bulb and changing the time when the current flows. The energy of the light bulb depends on the time of passing through the current. When the energy is relatively small, the average current of the light bulb is relatively low, showing that the brightness of the light bulb is darker; when the current flows through a longer time, the energy obtained by the light bulb is higher, showing that the light bulb is brighter, according to the research we know. The human eye is not sensitive to the flickering characteristics of the light. When the frequency reaches a certain value, the human eye will not notice the flickering of the light.

\subsection{Software Design}

The system software design mainly realizes the communication between STM32 host computer and infrared remote control, the communication between coordinator and node, and the control equipment of node. When the system is initialized, the network is formed by the coordinator, and the node joins the network and sends its address to the coordinator. The coordinator receives the node address and saves the node address. The curtains control interface layout is composed of a nested linear layout with relative layout. The main function of curtain control is curtain property and create two control, curtain property allows users to modify the name of the curtain freely. Curtain control function is mainly composed of linear layout nesting, gray graphics and buttons below constitute a vertical linear layout, three same linear layout horizontal arrangement constitutes the entire curtain control layout. In curtain control, the open, close and stop buttons are respectively bound to listeners. When the user clicks which button can make the circle on which button show yellow, which means that the button is selected, and the corresponding instructions are transmitted through the established Socket to control the rotation of the motor to achieve the control of the curtain.

The control function of temperature and humidity monitoring is realized by the mode of APP sending messages and MCU receiving messages and responding to them. This control page is implemented in a slightly different way. Temperature and humidity monitoring are to send messages to the WIFI module by clicking on the acquisition button. The WIFI module receives messages and then transmits them to the MCU. The MCU obtains information through the information acquisition module, and sends the information to APP through the WIFI module. The APP receives the data and displays it. The layout file is relatively simple, the whole is a vertical linear layout, the linear layout by three horizontal linear layouts, the first two linear layout is the same, but the content is different. The third linear layout is a humidifier switch, which enables humidifier control. The interface corresponding to the Java file, mainly for two access buttons to add listeners, when the user clicks, send information to the microcontroller, and receive the microcontroller return information, and then read and display in the position of the use. The humidifier switch is also bound to the listener. When the user clicks, the message is transmitted to the WIFI module, and a text box pops up to indicate whether the humidifier is open.

The way of gas detection is like temperature and humidity detection and control. Users can click the Access button when they want to see if the kitchen gas, mainly methane and natural gas, is in a safe range. The single chip microcomputer receives the message, obtains the data acquisition module information processing, returns the information, after judging, prompts the user whether is safe. Gas detection interface layout is relatively simple, consisting of a horizontal linear layout, similar to the temperature and humidity detection interface. Gas monitoring results are related to safety, so the return results are achieved by popping up a small window. This interaction can enhance people's awareness of vigilance. Small window through the new Alert Dialog class, by calling his Builder method to implement the specific, we want to prompt the user to set the message, and ultimately use the show () method to achieve. Binding the listener of the Alert Dialog class and the access control can achieve the corresponding functions. The next function is the timing task, which turns on and off the lights regularly. These two lines are implemented by a relative layout. The left side of the timing control is aligned with the parent control, the minute control and the parent control are aligned right, the left side of the intermediate input information and the timing control are aligned, and the right side 
and the minute control are aligned. Lighting patterns are also nested in different layouts, with a gray circle and buttons underneath a vertical linear layout. Three such layouts form a horizontal linear layout to achieve the layout of the lighting mode.

\section{Implementation of Intelligent Home Control System under the Concept of IoT}

This system mainly includes three parts: terminal management, log management and configuration management. Intelligent management system realizes the real-time monitoring of the status of household appliances terminal through the operation interface, as well as the functions of household appliances terminal control, abnormal recovery and historical playback. At the same time, to facilitate operation tracking and other purposes, the log management function is added to realize the query and setting of operation log. To add and delete terminals, connect configuration and system login account management functions, the system also designed the corresponding configuration management functions to facilitate the configuration of related parameters. The system is verified by analog access to three terminal sensors, namely washing machine, refrigerator and intelligent TV. In the actual test process, the system runs stably, can accurately detect sensor data, and realize intelligent switch control and state feedback of household appliances. Through abnormal scene simulation, intelligent exception handling can be effectively implemented. At the same time, the related mobile terminal can also receive abnormal alarm and status change message in time, which greatly reduces the manual intervention to achieve intelligent control. The lighting control module selects AT89C51 microcontroller as terminal control. When the external light is strong, the resistance value of photoresistor R13 is small, the level is low; when the external light is weak, the resistance value of photoresistor R13 is large, then the level is high, the level is sent to the microcontroller, and the program controls whether the illumination is realized. Smoke sensors can effectively detect the concentration of combustible or toxic gases in the environment, and detect hidden safety hazards in the home, to prevent fire or accidents. TGS813 gas sensor is selected to detect domestic flammable gases such as liquefied gas or natural gas. When abnormal scenes occur in household appliances, the sensor will send alarm information to the high-performance processor in time. The high-performance processor processes according to the preset intelligent processing scheme, and sends the abnormal alarm information to the remote mobile terminal. When the high-performance processor completes the corresponding intelligent processing scheme, the remote mobile terminal is informed according to the processing results, so that the user can understand the current progress. Considering the different home environment, the experimental data are collected under different degrees of occlusion. The experimental results show that the interference between the nodes is low, the system runs stably, and the communication results are accurate. The system fully meets the requirements of smart home monitoring and control.

\section{Conclusion}

After the system is designed and tested, the system runs stably, and the brightness of the lamp is adjusted and the switch of the fan and curtain is controlled accurately. The control system is simple in structure, accurate in control, convenient in expansion and low in cost. It is suitable for general family use and has high popularization value.

\section{References}

[1] Xun Yanli, Jiao Ku, Zhang Qinfei. Design and implementation of smart home control system based on IoT [J]. Modern Electronics Technique, 2018, 41(10): 74-76+80.

[2] Yu Xinhe, Zhang Haohua, Zhao Xiaoshu. Zigbee technology based smart home remote control system [J]. Journal of Shenyang Normal University (Natural Science Edition), 2015, 33(2): 281-284.

[3] Li Yu, Wang Weixing, Chen Runze. The Design Of Internet Of Things Smart Home System Based On ZigBee [J]. Electronic Test, 2016(5): 71-75. 
[4] Xia Huafeng, Xu Sheng. Study on Intelligent Control of Home System [J]. Industrial Control Computer, 2017(11): 88-89+92. 